\title{
Integrative analysis of miRNA-mRNA expression profiles in esophageal fibrosis after ESD
}

\author{
YIN ZHANG $^{1,2}$ and CHUNFANG XU ${ }^{1}$ \\ ${ }^{1}$ Department of Gastroenterology, The First Affiliated Hospital of Soochow University, Suzhou, Jiangsu 215006; \\ ${ }^{2}$ Department of Digestive Diseases, The First People's Hospital of Changzhou, \\ The Third Affiliated Hospital of Soochow University, Changzhou, Jiangsu 213003, P.R. China
}

Received October 18, 2020; Accepted March 1, 2021

DOI: $10.3892 /$ etm. 2021.10610

\begin{abstract}
The incidence of esophageal fibrosis and benign esophageal stricture (BES) has increased in recent years due to the curative therapy for early-stage esophageal carcinoma, including partial esophagectomy and esophageal endoscopic submucosal dissection (ESD). The aim of the present study was to identify key genes and associated pathways of esophageal fibrosis after the ESD procedure. During the esophageal ESD procedure, the esophageal tissue in the remaining submucosal layer, referred to as normal esophageal (NE) tissue, was collected, and 1 week thereafter, post-operative esophageal (PE) tissue was obtained. High-throughput sequencing was used to identify dysregulated microRNAs (miRNAs/miRs) between NE and PE tissues. According to the differentially expressed (DE) miRNAs, putative target genes were predicted. Gene Ontology, Kyoto Encyclopedia of Genes and Genomes enrichment analysis and DEmiRNA interaction network analysis were performed. Reverse transcription-quantitative PCR (RT-qPCR) was performed to validate the RNA-sequencing results. A total of 199 miRNAs were determined to be DE between NE and PE tissues. Compared with the expression in the NE group, 83 miRNAs were significantly upregulated, while 116 miRNAs were significantly downregulated. According to these DE miRNAs, forkhead box O1 (FOXO1), paired box 6 (PAX6), phosphatidylinositol-4,5-bisphosphate 3-kinase catalytic subunit alpha (PIK3CA) and adrenoceptor $\beta 1$ (ADRB1) were DE genes regulated by five DE miRNAs, including miR-223-3p, miR-142-5p, miR-582-5p, miR-21-3p and miR-218-5p. The results suggested that certain pathways were markedly dysregulated, including
\end{abstract}

Correspondence to: Professor Chunfang Xu, Department of Gastroenterology, The First Affiliated Hospital of Soochow University, 188 Shizi Street, Suzhou, Jiangsu 215006, P.R. China E-mail: xcf601@163.com

Key words: esophageal fibrosis, benign esophageal stricture, high-throughput sequencing, differentially expressed microRNAs, differentially expressed genes
FOXO, MAPK, AMP-activated protein kinase and signaling pathways regulating the pluripotency of stem cells and proteoglycans in cancer. According to the RT-qPCR results, the expression levels of FOXO1, PAX6, ADRB1, miR-223-3p, miR-582-5p, miR-21-3p and miR-218-5p were consistent with the integrated analysis. In conclusion, FOXO1, PAX6, PIK3CA and ADRB1 may have a role in esophageal fibrosis, regulated by miR-223-3p, miR-142-5p, miR-582-5p, miR-21-3p and $\mathrm{miR}-218-5 \mathrm{p}$. The present results provided an improved understanding of the changes in the microenvironment during the process of esophageal fibrosis, as well as novel potential targets for the treatment of esophageal fibrosis and BES.

\section{Introduction}

The incidence of benign esophageal stricture (BES) as a result of esophageal fibrosis has increased in recent years and is caused by a variety of esophageal injuries and inflammation, including partial esophagectomy, gastroesophageal reflux, caustic esophageal injury and radiotherapy (1-3). Screening programs of early detection of esophageal carcinoma have been implemented, which have led to earlier diagnosis and improved long-term success in patients with esophageal carcinoma. An increasing number of post-operative esophageal stricture cases require to be treated. The frequency of post-operative esophageal stricture ranges from 10 to $43 \%$ after esophagectomy $(4,5)$. In addition, the number of cases of BES after endoscopic submucosal dissection (ESD) has markedly increased over the past decade $(4,5)$. Although ESD is gradually becoming a standard treatment for early esophageal cancer, the post-operative complication of esophageal stricture cannot be ignored. The rate of post-operative esophageal stricture is $88-100 \%$ if the lesion measures $>3 / 4$ of a circumference (6). In the process of esophageal repair after ESD, fibroblasts produce extracellular matrix (ECM) and transform into myofibroblasts from 1 week after the resection. The latter causes wound contraction, which leads to scar formation and fibrosis. Thus, it is important to clarify the changes in the wound microenvironment $\sim 1$ week after ESD $(7,8)$. However, the prevention or treatment of BES remains difficult. The mechanisms of esophageal fibrosis and BES have remained largely elusive. Therefore, investigating the mechanism of esophageal fibrosis after ESD may provide approaches to develop novel treatments. 
MicroRNAs (miRNAs/miRs) are a series of small non-coding RNAs with a length of 20-24 nucleotides. It has been reported that miRNAs regulate $>60 \%$ of protein-coding genes by acting on mRNAs and then inhibiting or reducing their translation (9). Multiple miRNAs and mRNAs interact with each other (10). Of note, dysregulation of certain miRNAs and their target genes may contribute to the process of fibrosis. Identifying novel treatment strategies for BES prevention and treatment is essential. In postoperative epidural fibrosis tissue, mitomycin $\mathrm{C}$ (MMC) was able to reduce fibrosis by regulating miR-200b expression and targeting RhoE (11). In a study by Wang et al (12), microarray analysis of laminectomy scar tissue indicated that the expression levels of miR-34a, miR-146a and miR-200 were significantly increased, while the levels of miR-16, miR-221 and miR-378a were significantly decreased. Thus, it was speculated that various dysregulated miRNAs and their genes have a role in the process of esophageal fibrosis.

Through performing an integrated analysis, the present study aimed to identify the core miRNAs, differently expressed (DE) genes (DEGs) and their network, as well as to explore pathways associated with the pathogenesis of esophageal fibrosis.

\section{Materials and methods}

Participants and tissue collection. The present study was approved by the Ethics Committee of The First Affiliated Hospital of Soochow University and The First People's Hospital of Changzhou (Third Affiliated Hospital of Soochow University, Suzhou, China; approval no. 2020-100). All participants provided written informed consent. The samples were collected from January 2018 to December 2018. In the normal esophageal (NE) group, the remaining submucosal tissue at the edge of the lesion immediately after the ESD procedure was collected. In the post-operative esophageal (PE) group, the tissue was collected at the edge of the lesion 7 days after ESD. For each group, a total of five cases were enrolled in the study. The inclusion criteria were as follows: i) Patients diagnosed with esophageal high-grade intraepithelial neoplasia or early esophageal carcinoma prior to ESD and by post-operative pathology; and ii) the wound margin and base were both negative. The exclusion criteria were as follows: i) Patients $<18$ or $>80$ years of age; and ii) patients with coagulation dysfunction, abdominal aortic aneurysm and those who were not able to tolerate an endoscopic procedure. The characteristics of the participants are displayed in Table I. The tissue was obtained from the patients and immediately stored at $-80^{\circ} \mathrm{C}$ for further RNA extraction.

Microarray analysis of miRNAs. Total RNA was isolated from the tissue by using TRIzol reagent (Invitrogen; Thermo Fisher Scientific, Inc.). The concentration and purity of total RNA were determined using NanoDrop NC2000 spectrophotometer (Thermo Fisher Scientific, Inc.). RNA integrity was accessed by agarose gel electrophoresis. The microarray with the Agilent Human miRNA Microarray kit, Release 21.0, $8 \times 60 \mathrm{~K}$ (design ID: 070156) and data analysis of the 10 samples from esophageal submucosal tissues were performed by OE Biotechnology Co., Ltd. The miRNA array contained 2,570 probes. miRNA expression was evaluated with an Agilent Bioanalyzer 2100 (Agilent Technologies, Inc.). Total RNA was
Table I. Characteristics of the participants.

\begin{tabular}{lccc}
\hline Item & $\begin{array}{c}\text { NE group } \\
(\mathrm{n}=5)\end{array}$ & $\begin{array}{c}\text { PE group } \\
(\mathrm{n}=5)\end{array}$ & P-value \\
\hline $\begin{array}{l}\text { Male sex } \\
\text { Age (years) }\end{array}$ & 3 & 2 & $\mathrm{~ns}$ \\
$\begin{array}{l}\text { Postoperative pathology } \\
\text { High-grade }\end{array}$ & 3 & 4 & $\mathrm{~ns}$ \\
$\begin{array}{l}\text { intraepithelial neoplasia } \\
\begin{array}{l}\text { Early esophageal } \\
\text { carcinoma }\end{array}\end{array}$ & 2 & $66(57-70)$ & $\mathrm{ns}$ \\
\hline
\end{tabular}

Values are expressed as median (range) or n. NE, normal esophageal tissue; PE, esophageal samples taken at 1 week post-operatively; ns, not significant.

dephosphorylated, ligated with $\mathrm{pCpCy} 3$ and then hybridized to the microarray samples, which were then washed. Using an Agilent Scanner G2505C (Agilent Technologies, Inc.), the array was analyzed.

Data analysis. Raw data and array images were analyzed using Feature Extraction software (version 10.7.1.1; Agilent Technologies, Inc.) and GeneSpring software (version 14.8; Agilent Technologies, Inc.). The genes detected in all samples were further analyzed. DE miRNAs were selected based on fold change and P-value according to Student's t-test. Significant DEGs were considered those exhibiting a fold change $\geq 2.0$ and a $P \leq 0.05$. The target genes of these dysregulated miRNAs were predicted with two databases miRDB (http://www.mirdb.org/) and miRWalk (http://mirwalk.umm. uni-heidelberg.de/). Hierarchical clustering was performed to confirm the dysregulated miRNAs among samples between the two groups (NE and PE group).

miRNA-target prediction. In order to identify the key miRNA-mRNA interactions in esophageal fibrosis, target genes of the DE miRNAs were predicted using two databases (miRWalk and miRDB). The intersection of the two databases was considered. Those target genes that were predicted by $\geq 4$ algorithms were included in the study. miRNAs that reduced the target gene expression at the post-transcriptional level and the inversely correlated genes were selected as miRNA targets. The interaction network was built using Cytoscape software 3.7.2 (http://www. cytoscape.org/).

Gene Ontology (GO) and Kyoto Encyclopedia of Genes and Genomes (KEGG) analyses. To elucidate the functional roles of miRNAs and their target genes, $\mathrm{GO}$ (molecular function, biological process and cellular component) and KEGG pathway enrichment analyses were performed. A false discovery rate (FDR) $<0.05$ was considered to indicate a statistically significant difference.

Reverse transcription-quantitative PCR (RT-qPCR) validation of DE miRNAs and DEGs. RT-qPCR analysis was performed to verify the expression of the integrated analysis. Total RNA was isolated from the tissue which was the same as 
Table II. Primers used in reverse transcription-quantitative PCR.

\begin{tabular}{|c|c|}
\hline Name & Primer sequences $\left(5^{\prime}-3^{\prime}\right)$ \\
\hline $\operatorname{miR}-223-3 p$ & $\begin{array}{l}\text { F: GCCGAGACCCCAUAAACUG } \\
\text { R: CAGTGCGTGTCGTGGAGT }\end{array}$ \\
\hline $\operatorname{miR}-142-5 p$ & $\begin{array}{l}\text { F: CATAAAGTAGAAAGCACTACT } \\
\text { R: GCGAGCACAGAATTAATACGAC }\end{array}$ \\
\hline miR-582-5p & $\begin{array}{l}\text { F: GCGGTTACAGTTGTTCAACC } \\
\text { R: CTCAACTGGTGTCGTGGA }\end{array}$ \\
\hline $\operatorname{miR}-21-3 p$ & $\begin{array}{l}\text { F: ACGTCAACAGCAGTCGATGG } \\
\text { R: TATGGTTGTTCTGCTCTCTCTGTCTC }\end{array}$ \\
\hline miR-218-5p & $\begin{array}{l}\text { F: TGCGGCGGCCCCACGCACCAG } \\
\text { R: CCAGTGCAGGGTCCGAGGT }\end{array}$ \\
\hline FOXO1 & $\begin{array}{l}\text { F: AATCCAGAGGGTGGCAAGAGCG } \\
\text { R: GGCGAAATGTACTCCAGTTATCAA }\end{array}$ \\
\hline PAX6 & $\begin{array}{l}\text { F: CCCATGCAGATGCAAAAGTC } \\
\text { R: GCCAGTCTCGTAATACCTGC }\end{array}$ \\
\hline PIK3CA & $\begin{array}{l}\text { F: CCACGACCATCATCAGGTGAA } \\
\text { R: CCTCACGGAGATTCTAAAGT }\end{array}$ \\
\hline ADRB 1 & $\begin{array}{l}\text { F: GCTGCAGACGCTCACCA } \\
\text { R: GCGAGGTAGCGGTCCAG }\end{array}$ \\
\hline U6 & $\begin{array}{l}\text { F: CTCGCTTCGGCAGCACA } \\
\text { R: AACGCTTCACGAATTTGCGT }\end{array}$ \\
\hline$\beta$-actin & $\begin{array}{l}\text { F: TGGCACCCAGCACAATGAA } \\
\text { R: CTAAGTCATAGTCCGCCTAGAA }\end{array}$ \\
\hline
\end{tabular}

FOXO1, forkhead box O1; PAX6: paired box 6; PIK3CA, phosphatidylinositol-4,5-bisphosphate 3-kinase catalytic subunit alpha; ADRB 1 , adrenoceptor $\beta 1 ; \mathrm{F}$, forward; R, reverse.

the microarray. Total RNA (400 ng) was converted to cDNA using TransScript All-in-One First-Strand cDNA Synthesis SuperMIX (cat. no. AT341-01; Beijing Transgen Biotech Co., Ltd.) following the manufacturer's instructions. The reverse transcription temperature protocols were $42^{\circ} \mathrm{C}$ for $15 \mathrm{~min}, 85^{\circ} \mathrm{C}$ for $5 \mathrm{sec}$ and maintained at $4^{\circ} \mathrm{C}$. The final products were stored at $-20^{\circ} \mathrm{C}$. qPCR was conducted with Takara SYBR Premix Ex Taq $^{\mathrm{TM}}$ (Tli RNaseH Plus; Takara Bio, Inc.) in a 7900HT Fast Real-Time PCR system (Thermo Fisher Scientific, Inc.). The primer sequences are showed in Table II. The reaction procedure consists of 40 cycles: Initial denaturation at $94^{\circ} \mathrm{C}$ for $30 \mathrm{sec}$, followed by 40 cycles of $94^{\circ} \mathrm{C}$ for $5 \mathrm{sec}$ and $60^{\circ} \mathrm{C}$ for $30 \mathrm{sec}$. U6 was used as endogenous control for miRNAs and $\beta$-actin was for mRNAs. The relative expression values were calculated using the $2-\Delta \Delta \mathrm{Cq}$ method (13).

Statistical analysis. Statistical analysis was performed using SPSS 19.0 software (SPSS, Inc.). Values are expressed as the mean \pm standard deviation (relative expression of RNAs) or median (range) age. The unpaired Student's t-test was used to compare parameter variables (relative expression of RNAs, age). Fisher's exact test was used to compare categorical variables (male sex, postoperative pathology). $\mathrm{P}<0.05$ was considered to indicate a statistically significant difference.

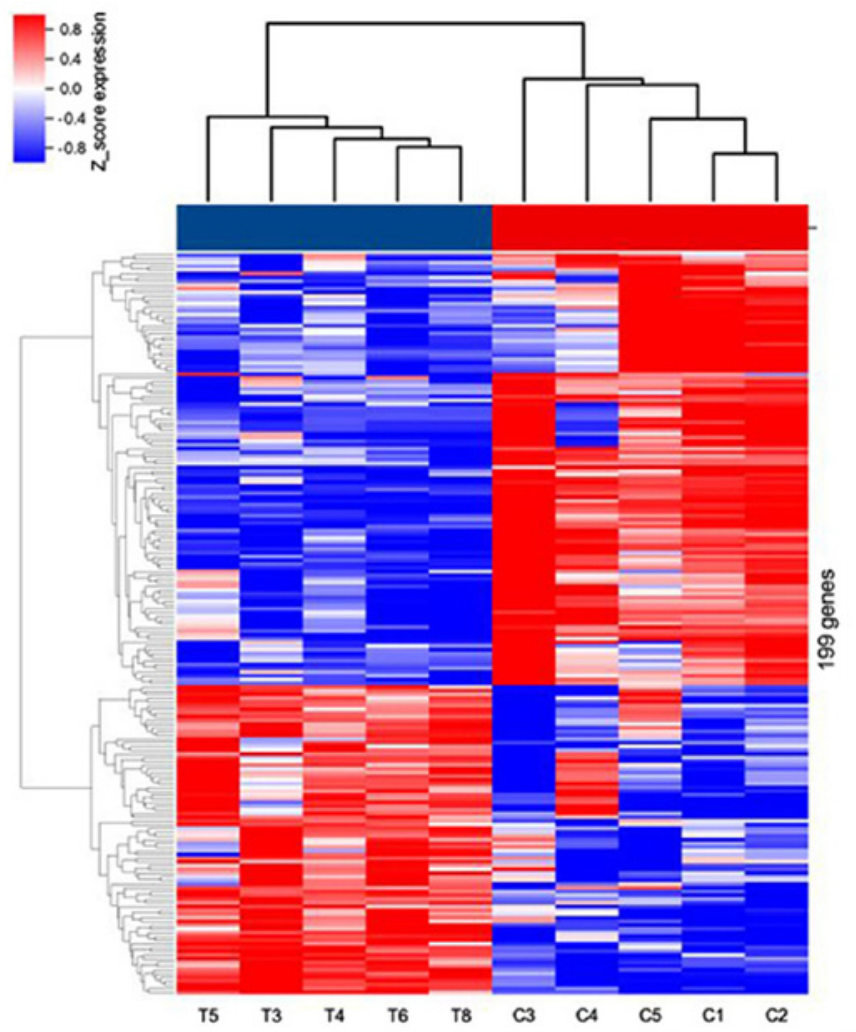

Figure 1. Unsupervised hierarchical clustering identification of two distinct groups (normal esophageal vs. post-operative esophageal group) based on their miRNA expression profile. Sample names are listed at the top. The 199 miRNAs in the heat map were differentially expressed $(\mathrm{P}<0.05)$ according to the results of the analysis of target genes. miRNA, microRNA.

\section{Results}

Differential expression analysis of genes in esophageal fibrosis. Using miRNA microarray analysis, numerous transcripts were detected in PE and NE tissues. In total, 199 miRNAs were significantly dysregulated with fold change $>2.0, \mathrm{P}<0.05$ and FDR $<0.05$. Among them, 83 miRNAs were upregulated, while 116 miRNAs were downregulated. The dysregulated miRNAs are displayed in a heat-map (Fig. 1). The top 10 upregulated and downregulated miRNAs are listed in Table III.

GO and KEGG enrichment analyses of dysregulated miRNAs. GO and KEGG pathway enrichment analyses were performed to explore the pathogenesis of esophageal fibrosis. In the $\mathrm{GO}$ enrichment analysis, the main affected biological processes included positive regulation of transcription mediated by the RNA polymerase II promoter, negative regulation of transcription mediated by the RNA polymerase II promoter, positive regulation of transcription (DNA template), nervous system development and transcription mediated by the RNA polymerase II promoter. The cellular component terms included cytosol, nucleoplasm, cytoplasm, neuron projection and growth cone. Molecular functions mainly involved protein binding, transcriptional activator activity, RNA polymerase II core promoter proximal region sequence-specific binding, protein serine/threonine kinase activity, $\beta$-catenin binding and mRNA 3'-untranslated region (UTR) binding (Fig. 2). 


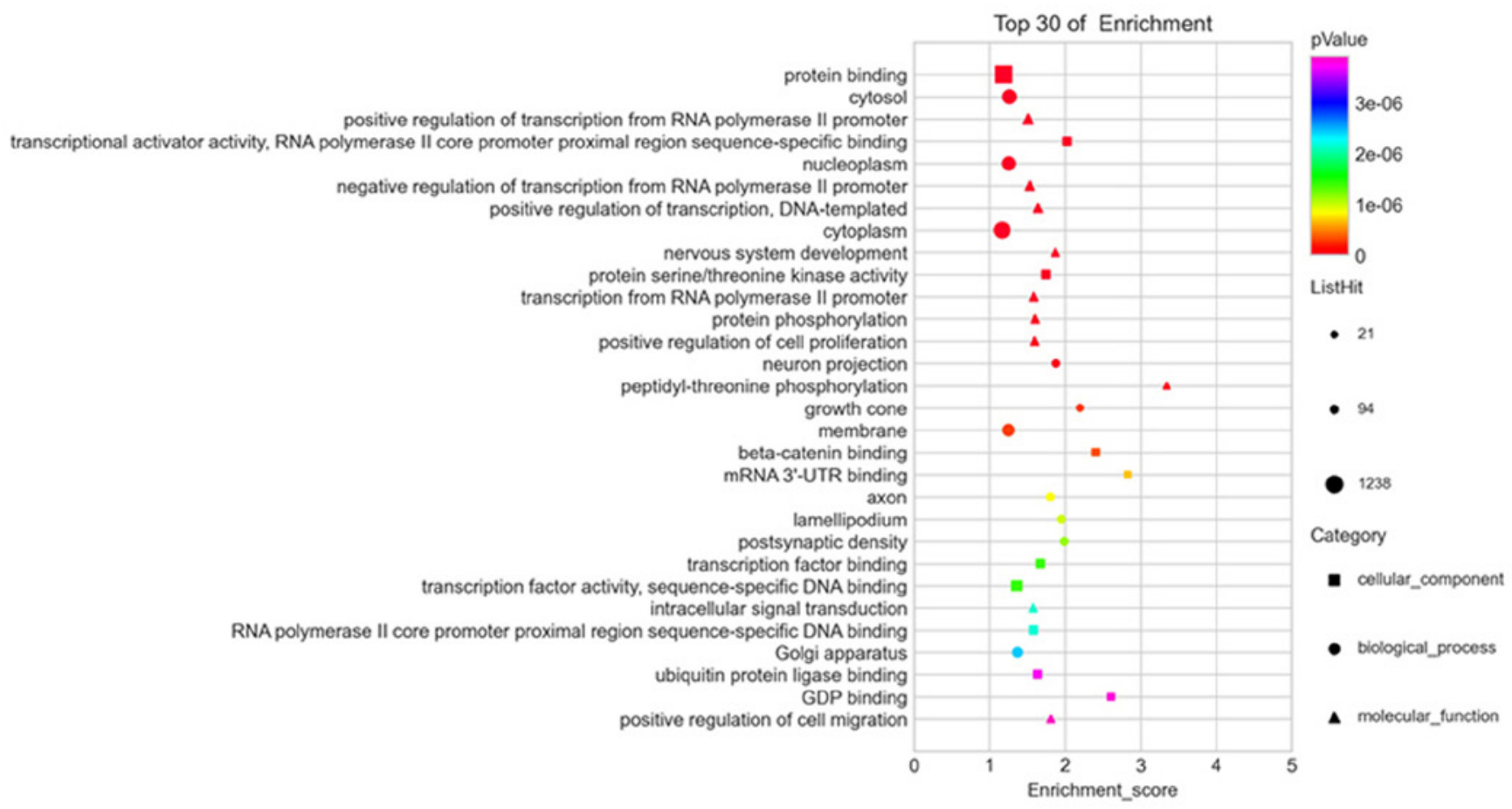

Figure 2. GO analysis of differentially expressed microRNAs. GO analysis according to biological process, molecular function and cellular component, ranked by enrichment score $[-\log 10$ (P-value)]. GO, Gene Ontology.

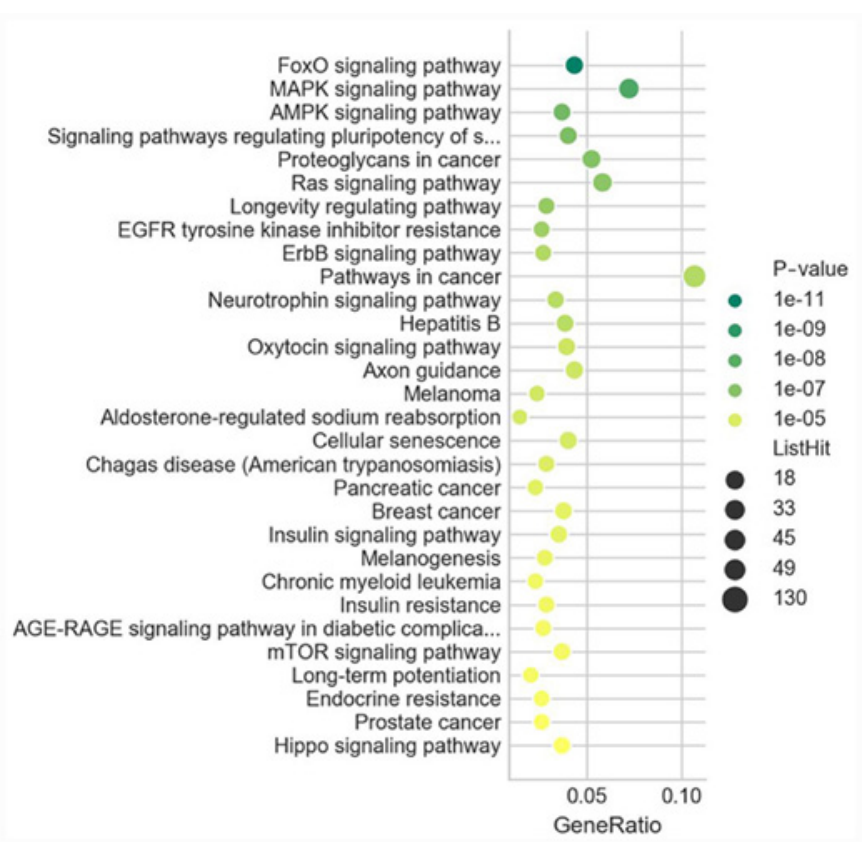

Figure 3. Kyoto Encyclopedia of Genes and Genomes analysis of differentially expressed microRNAs ranked by enrichment score [-log10 (P-value)]. FoxO, Forkhead Box O; AMPK, AMP-activated protein kinase; EGFR, epidermal growth factor receptor; AGE-RAGE, advanced glycation end product-receptor for AGE.

The results of the KEGG pathway enrichment analysis revealed the core pathways involved in esophageal fibrosis, including the forkhead box O (FOXO), MAPK and AMP-activated protein kinase (AMPK) signaling pathways, as well as signaling pathways regulating the pluripotency of stem cells and proteoglycans in cancer (Fig. 3).

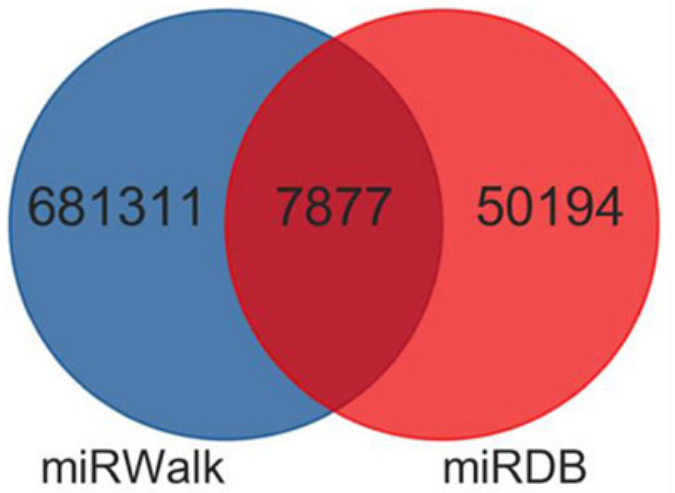

Figure 4. miRNA-target prediction using two databases (miRWalk and miRDB). Blue and red colors represent target genes predicted by the databases miRWalk and miRDB, respectively. miR/miRNA, microRNA.

Target genes predicted by DE miRNAs and miRNA-mRNA interaction network. A total of 7,878 miRNA-mRNA interaction pairs were obtained, of which 2,745 miRNA-mRNA pairs were upregulated and 5,133 miRNA-mRNA pairs downregulated (Fig. 4). According to these pairs, the miRNA-mRNA interaction network was built. The network consisted of the top 10 related dysregulated miRNAs (miR-218-5p, miR-345-5p, miR-424-3p, miR-1246, miR-142-5p, miR-21-3p, miR-223-3p, miR-223-5p, miR-582-5p and miR-605-5p) and 143 predicted DEGs (Fig. 5). In total, five miRNAs (miR-223-3p, miR-142-5p, miR-582-5p, miR-21-3p and miR-218-5p) were selected as core DE miRNAs that interacted with the majority of DEGs according to the interaction network analysis, and the GO and KEGG analyses. According to the DE miRNAs obtained, four predicted DEGs [FOXO1, paired box 6 (PAX6), phosphatidylinositol-4,5-bisphosphate 3-kinase catalytic 


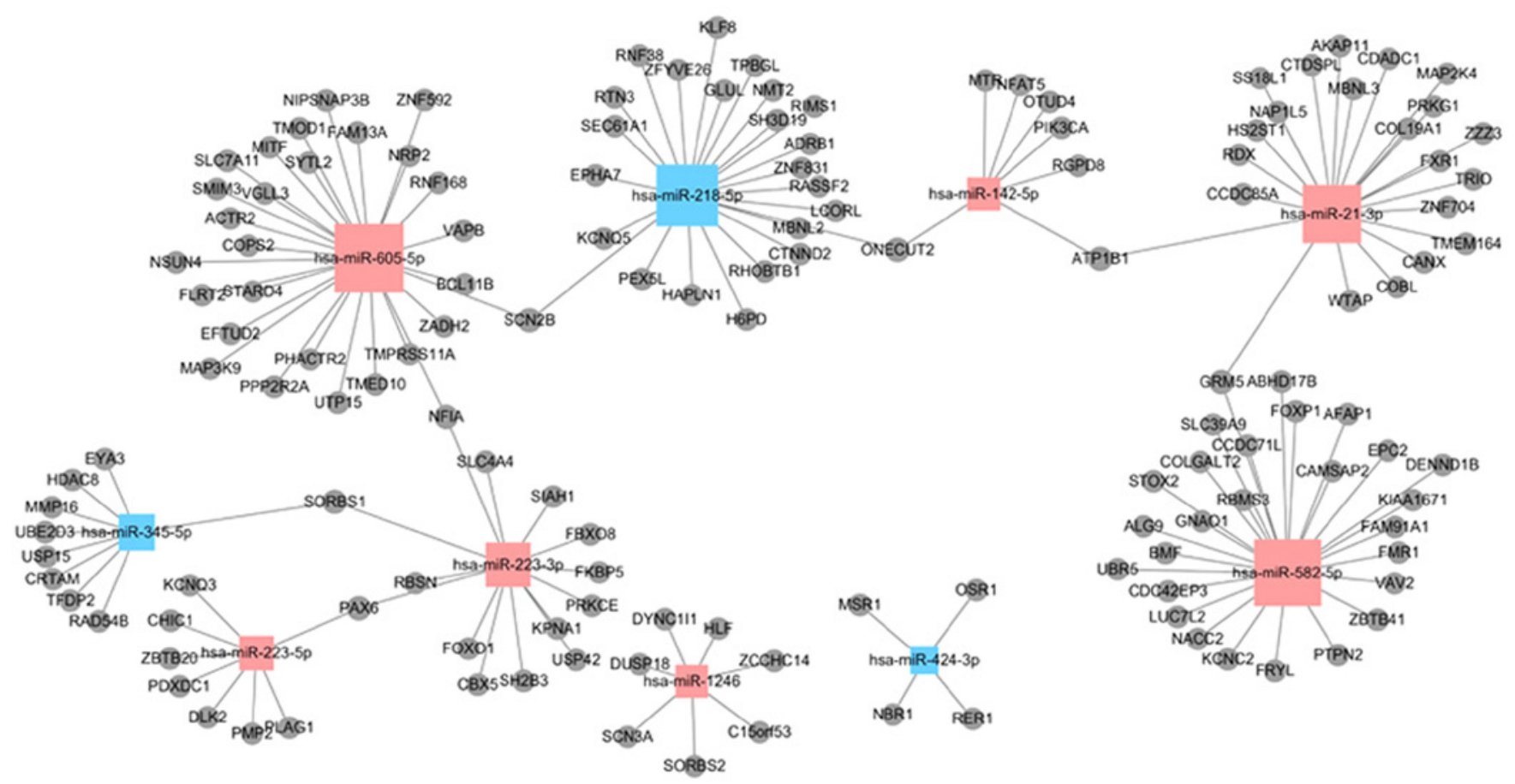

Figure 5. Esophageal fibrosis-specific differentially expressed miR-mRNA regulatory network. Red represents upregulation and blue represents downregulation. miR, microRNA; hsa, Homo sapiens.)

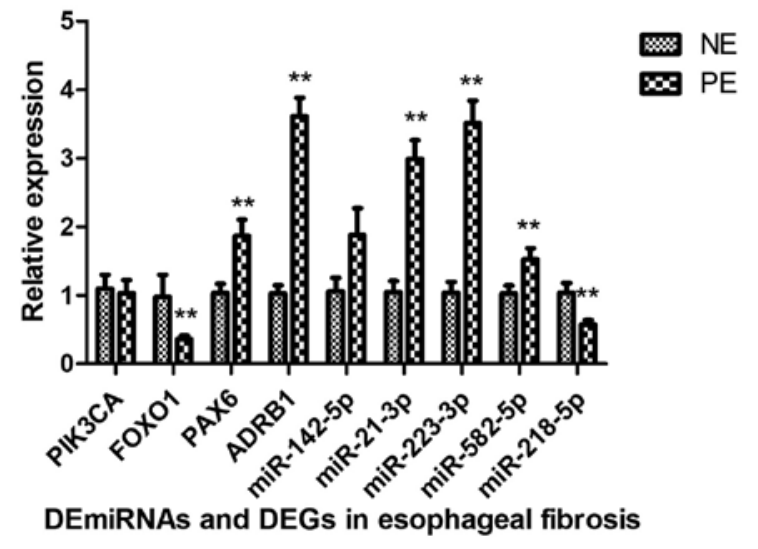

Figure 6. Reverse transcription-quantitative PCR results of DE micRNAs and target DE genes in esophageal fibrosis. DE, differentially expressed; miRNA/miR, microRNA; NE, normal esophageal tissue; PE, esophageal samples taken at 1 week post-operatively; PIK3CA, phosphatidylinositol-4,5-bisphosphate 3-kinase catalytic subunit alpha; FOXO1, forkhead box O1; ADRB1, adrenoceptor $\beta 1$; PAX6, paired box 6 . ( ${ }^{* *} \mathrm{P}<0.05$ vs. NE group).

subunit alpha (PIK3CA) and adrenoceptor $\beta 1$ (ADRB1)] were selected, which were also involved in the process of fibrosis.

Verification of expression changes in miRNAs and mRNAs. To confirm whether the genes were consistent with the integrated analysis, five DE miRNAs (miR-223-3p, miR-142-5p, miR-582-5p, miR-21-3p and miR-218-5p) and four targets (FOXO1, PAX6, PIK3CA and ADRB1) were selected. The results revealed that FOXO1 and miR-218-5p were downregulated, while miR-223-3p, miR-582-5p, miR-21-3p, PAX6 and ADRB1 were upregulated. However, the expression of PIK3CA and miR-142-5p was inconsistent with the results of the integrated analysis (Fig. 6).
Table III. Top 10 overexpressed and top 10 downregulated miRNAs in esophageal samples taken at 1 week post-operatively vs. normal esophageal samples.

\begin{tabular}{|c|c|c|c|c|}
\hline Probe ID & P-value & $\mathrm{FC}$ & $\log 2 \mathrm{FC}$ & $\begin{array}{c}\text { Direction of } \\
\text { regulation }\end{array}$ \\
\hline hsa-miR-223-3p & $2.75 \times 10^{-5}$ & 64.46 & 6.01 & Up \\
\hline hsa-miR-3663-5p & $1.94 \times 10^{-5}$ & 48.76 & 5.61 & Up \\
\hline hsa-miR-223-5p & $1.84 \times 10^{-4}$ & 36.43 & 5.19 & Up \\
\hline hsa-miR-142-5p & $4.40 \times 10^{-4}$ & 35.13 & 5.13 & Up \\
\hline hsa-miR-31-3p & 0.022 & 30.50 & 4.93 & Up \\
\hline hsa-miR-605-5p & $2.38 \times 10^{-4}$ & 27.57 & 4.79 & Up \\
\hline hsa-miR-4451 & $2.81 \times 10^{-6}$ & 24.63 & 4.62 & Up \\
\hline hsa-miR-1246 & $1.56 \times 10^{-7}$ & 16.45 & 4.04 & Up \\
\hline hsa-miR-582-5p & $9.05 \times 10^{-3}$ & 13.71 & 3.78 & Up \\
\hline hsa-miR-21-3p & $4.53 \times 10^{-4}$ & 13.17 & 3.72 & Up \\
\hline hsa-miR-4647 & $7.84 \times 10^{-5}$ & -58.61 & -5.87 & Down \\
\hline hsa-miR-6129 & $3.89 \times 10^{-6}$ & -46.88 & -5.55 & Down \\
\hline hsa-miR-4522 & $4.10 \times 10^{-5}$ & -43.70 & -5.45 & Down \\
\hline hsa-miR-1273c & $3.93 \times 10^{-7}$ & -40.00 & -5.32 & Down \\
\hline hsa-miR-4446-3p & $9.54 \times 10^{-7}$ & -38.85 & -5.28 & Down \\
\hline hsa-miR-218-5p & $5.03 \times 10^{-3}$ & -36.99 & -5.21 & Down \\
\hline hsa-miR-5699-5p & $1.62 \times 10^{-4}$ & -34.97 & -5.13 & Down \\
\hline hsa-miR-424-3p & $4.28 \times 10^{-7}$ & -33.47 & -5.06 & Down \\
\hline hsa-miR-345-5p & $6.97 \times 10^{-6}$ & -31.75 & -4.99 & Down \\
\hline hsa-miR-6857-5p & $9.26 \times 10^{-7}$ & -26.64 & -4.748 & Down \\
\hline
\end{tabular}

Hsa, Homo sapiens; miRNA/miR, microRNA; FC, fold change. 


\section{Discussion}

Studies have indicated that miRNAs have a vital role in the process of fibrosis, including myocardial, pulmonary and liver fibrosis (10-12). However, to the best of our knowledge, no previous studies have reported on the function of miRNAs in the pathobiology of esophageal fibrosis and subsequent BES. In the clinic, the prevention or treatment of BES remain challenging and identifying novel strategies for the prevention or treatment of esophageal fibrosis is of great importance. In the present study, miRNA microarray analysis was performed for evaluating DE miRNA expression associated with esophageal fibrosis. Compared with those in the NE control group, 83 overexpressed miRNAs and 116 downregulated miRNAs were identified in the patients 1 week after the esophageal ESD procedure. According to the results of GO and KEGG analyses, as well as the interaction network, four DEGs (FOXO1, PAX6, PIK3CA and ADRB1) and five DE miRNAs (miR-223-3p, miR-142-5p, miR-582-5p, miR-21-3p and miR-218-5p) were associated with esophageal fibrosis.

Regarding the collected tissue, the process of esophageal fibrosis after ESD, in which the mucosa and majority of the submucosal layer were dissected, were evaluated (6). The majority of fibroblasts and fibrocytes are located in the connective tissue (submucosal or serosa layer) of the esophagus. Thus, after ESD, the surrounding fibroblasts are activated and participate in the process of esophageal fibrosis mainly in the remaining submucosal layer (7). In addition, in the process of esophageal repair after ESD, fibroblasts produce ECM and transform into myofibroblasts from 1 week after the resection. Thus, the remaining submucosal tissue after the ESD procedure was harvested immediately and 7 days thereafter.

FOXO1, a potential target gene of miR-223-3P, which usually has a key role in cell function, is a member of the FOX transcription factor family and was reported to participate in cell metabolism, proliferation, migration, apoptosis and angiogenesis progression (14). In a study by Han et al (15), miR-223-3p was indicated to be highly expressed in neuroblastoma cell lines as compared with its expression in normal cell lines, and it was able to bind to the 3'-UTR of FOXO1, thus resulting in a reduction in FOXO1 expression. The results revealed that miR-223-3p has an oncogenic role by promoting cell proliferation and invasion, and inhibiting apoptosis via targeting FOXO1. In other studies, miR-223 was also dysregulated in skeletal muscle and cardiac fibroblasts and acted as a fibrogenic factor $(16,17)$. In the present study, miR-223-3p and miR-223-5p were highly expressed and may serve as therapeutic targets. However, whether they are associated only with inflammation or with the process of esophageal fibrosis was not confirmed. The precise role of miR-223 requires further investigation.

PAX6 is a member of the PAX family of transcription factors and has been indicated to have essential roles in multiple organs and cell lines, including the eyes, central nervous system, pancreatic islet cells and olfactory system. It has been reported that PAX6 acted as an anti-fibrotic agent for cardiac fibroblast differentiation and cystic fibrosis and repressed the expression of the pro-fibrotic factor TGF- $\beta 1$ (18). Interference against PAX6 also inhibited the activation and proliferation of hepatic stellate cells (HSCs). Thus, PAX6 upregulation may induce HSC activation and proliferation, resulting in liver fibrosis (19). Based on these results, future studies should explore the fibrogenic effect of PAX6 on esophageal fibrosis.

ADRB1 is expressed in the apical membrane of human bronchial ciliated epithelia and participates in the process of pulmonary cystic fibrosis (20). Kiriazis et al (21) investigated the effect of ADRB1 on cardiac hypertrophic growth and fibrosis. The results also revealed the key role of $\beta$-adrenoceptor signaling and that ADRB1 may be an alternative therapeutic target for cardiac hypertrophy and fibrosis. The study by Ito et al (22) suggested that DRB1 has an important role in myocyte apoptosis and fibrosis in cardiac muscle and skeletal muscle. In the present study, ADRB1 was one of the target mRNAs of miR-218-5p, which may also be a regulator of the process of fibrosis.

Steiner et al (23) indicated that PIK3CA mutation leads to abnormal scarring involving the PI3K-Akt-mTOR pathway. Yang et al (24) reported that PIK3CA regulated myocardial fibrosis and oxidative stress via the PI3K/Akt signaling pathway. Wegner et al (25) indicated that PIK3CA and TGF- $\beta 1$ contributed to prostatic stromal hypertrophy and collagen accumulation. However, in the present study, the RT-qPCR results for PIK3CA revealed no significant difference between the two groups, whilst PIK3CA was negatively correlated with miR-142-5p, which was predicted with two databases miRDB and miRWalk. In other studies, PIK3CA was also negatively regulated by miR-142-5p in multiple tumors $(26,27)$. It may be speculated that this inconsistency may result from the small size of the samples and their heterogeneity. Thus, whether PIK3CA regulates esophageal fibroblast activation requires further investigation.

In cardiac fibrosis after myocardial infarction, miR-142 contributed to cardiac fibroblast activation by targeting the adenomatous polyposis coli and the subsequent Wnt signaling pathway (28). In idiopathic pulmonary fibrosis, miR-142-3p had a protective role against the pro-fibrotic effect of TGF- $\beta 1$ (29). In the process of non-small cell lung cancer, upregulated miR-142-5p suppressed cell proliferation by regulating PIK3CA (27). Together with the present results, the above suggested that miR-142 may have key roles in inflammation and fibrosis by regulating the expression of PIK3CA. However, the RT-qPCR results for miR-142-5p indicated no significant difference. In the microarray results, the miR-142 expression in the majority of patients with PE was higher than that in the NE group. However, there was excessive heterogeneity among different samples. In addition, a previous study suggested that miR-142-5p regulates PIK3CA and has a role in fibrosis (27). Thus, whether miR-142-5p and PIK3CA are involved in the process of esophageal fibrosis requires further evaluation.

miR-582-5p has also been revealed to be closely associated with inflammatory and immune response signaling in previous studies. It was reported to have a key role in the development of chronic inflammation of the airway and subsequent airway remodeling (30). In the cirrhotic liver, hepatic cytochrome P4503A (CYP3A) activity was determined to be decreased and miR-582-5p was highly expressed, which suggested that miR-582-5p expression may regulate hepatic CYP3A activity and liver cirrhosis (31). 
High expression of miR-21 has been determined in multiple fibrotic tissues. As reported by Yang et al (32), miR-21 contributed to the development of hepatic fibrosis via the TGF- $\beta / \mathrm{Smad} 7$ signaling pathway. miR-21 also regulated myofibroblast transformation and myocardial fibrosis. TGF- $\beta$ promoted cardiac fibroblast to myofibroblast transformation and myocardial fibrosis by upregulating miR-21, which in turn sponged the target mRNA Jagged1 (33). Furthermore, miR-21 was also reported to be a fibrosis-associated miRNA that promoted inflammation in ligamentum flavum tissue by activating IL-6 expression, leading to ligamentum flavum fibrosis and hypertrophy (34). In the present study, the expression level of miR-21-3p was significantly increased in the PE group, which is consistent with other studies (32-34).

miR-218-5p was reported to be significantly downregulated in smokers with chronic obstructive pulmonary disease compared with its expression in never-smokers. It was mostly highly expressed in the bronchial airway epithelium and was associated with airway obstruction (35). Li et al (36) and Brkić et al (37) indicated that miR-218-5p regulated the proliferation, migration and EMT of human glioma cells. Pterygium is a chronic ocular inflammatory disease that may result in various ocular surface symptoms. Upregulated miR-218-5p significantly suppressed the expression of epidermal growth factor receptor via the PI3K/Akt/mTOR pathway in epithelial cells affected by pterygium (38). As the results of the present study, miR-218-5p was downregulated in the PE group, suggesting that its overexpression may counteract the fibrotic process.

According to the KEGG enrichment analysis, the PIK3CA, FOXO, MAPK and AMPK pathway was among the top signaling pathways. These pathways have all been reported as key signaling pathways in the activation of fibrosis, which supports the present results $(23-25,15,39,40)$.

In the clinic, certain drugs may be used to prevent or treat esophageal stricture, including topical use of corticosteroids and mitomycin C (MMC) $(41,42)$. In a previous study by our group, topical injection of MMC was able to release the post-ESD esophageal stricture (42). However, the mechanisms of esophageal fibrosis have remained elusive, to the best of our knowledge. Only MMC was reported to reduce epidural fibrosis by regulating miR-200b expression and the target gene RhoE (11). In a study by Wang et al (12), after MMC treatment of laminectomy scar tissue, the expression levels of miR-34a, miR-146a and miR-200 were significantly increased, while the levels of miR-16, miR-221 and miR-378a were significantly decreased. However, whether these genes are differentially expressed during the process of esophageal fibrosis remains unclear. Thus, future studies should aim to validate the associations of the aforementioned drugs and these DE miRNAs and DE mRNAs.

The present study has several limitations. First, the post-ESD esophageal stricture model is not suitable for other types of esophageal fibrosis such as esophagectomy, as the mucosa and majority of submucosal layer were dissected during the ESD procedure, but the lamina propria and serosa remained intact. However, to the best of our knowledge, there is no animal model or study referring to the mechanism of esophageal stricture resulting from esophagectomy. However, all BES types are the result of esophageal fibrosis due to myofibroblast contraction and substantial deposition of ECM components. Thus, it may be partially confirmed which factors determine the process of esophageal fibrosis. In addition, only five cases were recruited in each group. Furthermore, the tissue was collected by endoscopic biopsy, which features broad heterogeneity. Thus, further studies with larger sample sizes are required.

In conclusion, using high-throughput sequencing, DE miRNAs and their DEGs were detected in the process of esophageal fibrosis. According to the RT-qPCR results, the expression levels of FOXO1, PAX6, ADRB1, miR-223-3p, miR-582-5p, miR-21-3p and miR-218-5p were consistent with the integrated analysis, but PIK3CA and miR-142-5p could not be confirmed. These reasons may include the large heterogeneity of patients and the false positive chip results. Furthermore, miR-223-3p may participate in esophageal fibrosis by targeting FOXO1 and PAX6. miR-218-5p also has a promoting role by targeting ADRB1. miR-142-5p may facilitate fibrosis by regulating PIK3CA. In addition, miR-582-5p and miR-21-3p also had roles in esophageal fibrosis. Further studies are required to functionally characterize the role of these candidate RNAs. The current results may enhance the understanding of the microenvironmental changes in the process of esophageal fibrosis and may provide targets for novel treatment strategies for esophageal fibrosis and BES.

\section{Acknowledgements}

Not applicable.

\section{Funding}

The study was partially funded by the National Natural Science Foundation of China (grant no. 81700575), the Young Talent Development Plan of Changzhou Health Commission (grant no. CZQM2020017), the Natural Science Foundation of the Jiangsu Higher Education Institutions of China (grant no. 18KJB320018) and the Primary Research \& Social Development Plan of Jiangsu Province (grant no. BE2018659).

\section{Availability of data and materials}

The datasets generated and/or analyzed during the current study are available in the Gene Expression Omnibus (accession no. GSE169354) repository (https://www.ncbi.nlm.nih. gov/geo/query/acc.cgi?acc=GSE169354).

\section{Authors' contributions}

CX conceived and designed the study, authenticated the raw data and performed manuscript review. YZ developed the methodology, performed the experiments, analyzed the data and wrote the manuscript. Both authors read and approved the final version of this manuscript.

\section{Ethics approval and consent to participate}

The present study was approved by the Ethics Committee of The First Affiliated Hospital of Soochow University and The First People's Hospital of Changzhou (Third Affiliated 
Hospital of Soochow University, Suzhou, China; approval no. 2020-100). All participants provided written informed consent.

\section{Patient consent for publication}

Not applicable.

\section{Competing interests}

The authors declare that they have no competing interests.

\section{References}

1. Siersema PD: How to approach a patient with refractory or recurrent benign esophageal stricture. Gastroenterology 156 7-10, 2019

2. Yan X, Nie D, Zhang Y, Chang H and Huang Y: Effectiveness of an orally administered steroid gel at preventing restenosis after endoscopic balloon dilation of benign esophageal stricture. Medicine (Baltimore) 98: e14565, 2019.

3. Fuccio L, Hassan C, Frazzoni L, Miglio R and Repici A: Clinical outcomes following stent placement in refractory benign esophageal stricture: A systematic review and meta-analysis. Endoscopy 48: 141-148, 2016.

4. van Halsema EE, Noordzij IC, van Berge Henegouwen MI, Fockens P, Bergman JJ and van Hooft JE: Endoscopic dilation of benign esophageal anastomotic strictures over $16 \mathrm{~mm}$ has a longer lasting effect. Surg Endosc 31: 1871-1881, 2017.

5. Haverkamp L, van der Sluis PC, Verhage RJ, Siersema PD, Ruurda JP and van Hillegersberg R: End-to-end cervical esophagogastric anastomoses are associated with a higher number of strictures compared with end-to-side anastomoses. J Gastrointest Surg 17: 872-876, 2013.

6. Liao Z, Liao G, Yang X, Peng X, Zhang X, Xie X, Zhao X, Yang S, Fan $\mathrm{C}$ and Bai J: Transplantation of autologous esophageal mucosa to prevent stricture after circumferential endoscopic submucosal dissection of early esophageal cancer (with video) Gastrointest Endosc 88: 543-546, 2018.

7. Rockey DC, Bell PD and Hill JA: Fibrosis - a common pathway to organ injury and failure. N Engl J Med 372: 1138-1149, 2015.

8. Honda M, Nakamura T, Hori Y, Shionoya Y, Nakada A, Sato T, Yamamoto K, Kobayashi T, Shimada H, Kida N, et al: Process of healing of mucosal defects in the esophagus after endoscopic mucosal resection: Histological evaluation in a dog model. Endoscopy 42: 1092-1095, 2010.

9. Yates LA, Norbury CJ and Gilbert RJ: The long and short of microRNA. Cell 153: 516-519, 2013.

10. Blaya D, Coll M, Rodrigo-Torres D, Vila-Casadesús M, Altamirano J, Llopis M, Graupera I, Perea L, Aguilar-Bravo B, Díaz A, et al: Integrative microRNA profiling in alcoholic hepatitis reveals a role for microRNA-182 in liver injury and inflammation. Gut 65: 1535-1545, 2016.

11. Sun Y, Ge Y, Fu Y, Yan L, Cai J, Shi K, Cao X and Lu C: Mitomycin $\mathrm{C}$ induces fibroblasts apoptosis and reduces epidural fibrosis by regulating miR-200b and its targeting of RhoE. Eur J Pharmacol 765: 198-208, 2015.

12. Wang BB, Xie H, Wu T, Xie N, Wu J, Gu Y, Tang F and Liu J: Controlled-release mitomycin C-polylactic acid film prevents epidural scar hyperplasia after laminectomy by inducing fibroblast autophagy and regulating the expression of miRNAs Eur Rev Med Pharmacol Sci 21: 2526-2537, 2017.

13. Livak KJ and Schmittgen TD: Analysis of relative gene expression data using real-time quantitative PCR and the 2(-Delta Delta C(T)) method. Methods 25: 402-408, 2001.

14. Wu L, Li H, Jia CY, Cheng W, Yu M, Peng M, Zhu Y, Zhao Q, Dong YW, Shao K, et al: MicroRNA-223 regulates FOXO1 expression and cell proliferation. FEBS Lett 586: 1038-1043, 2012.

15. Han LL, Zhou XJ, Li FJ, Hao XW, Jiang Z, Dong Q and Chen X: MiR-223-3p promotes the growth and invasion of neuroblastoma cell via targeting FOXO1. Eur Rev Med Pharmacol Sci 23. 8984-8990, 2019.

16. Cheng N, Liu C, Li Y, Gao S, Han YC, Wang X, Du J and Zhang C: MicroRNA-223-3p promotes skeletal muscle regeneration by regulating inflammation in mice. J Biol Chem 295: 10212-10223, 2020.
17. Liu X, Xu Y, Deng Y and Li H: MicroRNA-223 regulates cardiac fibrosis after myocardial infarction by targeting RASA1. Cell Physiol Biochem 46: 1439-1454, 2018.

18. Chen YT, Chen FY, Vijmasi T, Stephens DN, Gallup M and McNamara NA: Pax6 downregulation mediates abnormal lineage commitment of the ocular surface epithelium in aqueous-deficient dry eye disease. PLoS One 8: e77286, 2013.

19. Feng Y, Li M, Wang S, Cong W, Hu G, Song Y, Xiao H, Dong E and Zhang Y: Paired box 6 inhibits cardiac fibroblast differentiation. Biochem Biophys Res Commun 528: 561-566, 2020.

20. Li C, Tan YH, Sun J, Deng FM and Liu YL: PAX6 contributes to the activation and proliferation of hepatic stellate cells via activating Hedgehog/GLI1 pathway. Biochem Biophys Res Commun 526: 314-320, 2020

21. Kiriazis H, Wang K, Xu Q, Gao XM, Ming Z, Su Y, Moore XL, Lambert G, Gibbs ME, Dart AM, et al: Knockout of beta(1)- and beta(2)-adrenoceptors attenuates pressure overload-induced cardiac hypertrophy and fibrosis. Br J Pharmacol 153: 684-692, 2008.

22. Ito A, Ohnuki Y, Suita K, Ishikawa M, Mototani Y, Shiozawa K, Kawamura N, Yagisawa Y, Nariyama M, Umeki D, et al: Role of $\beta$-adrenergic signaling in masseter muscle. PLoS One 14: e0215539, 2019.

23. Steiner JE, Cottrell CE, Streicher JL, Jensen JN, King DM, Burrows PE, Siegel DH, Tollefson MM, Drolet BA and Püttgen KB: Scarring in patients With PIK3CA-related overgrowth syndromes. JAMA Dermatol 154: 452-455, 2018.

24. Yang X, Li X, Lin Q and Xu Q: Up-regulation of microRNA-203 inhibits myocardial fibrosis and oxidative stress in mice with diabetic cardiomyopathy through the inhibition of PI3K/Akt signaling pathway via PIK3CA. Gene 715: 143995, 2019.

25. Wegner KA, Mueller BR, Unterberger CJ, Avila EJ, Ruetten H, Turco AE, Oakes SR, Girardi NM, Halberg RB, Swanson SM, et al: Prostate epithelial-specific expression of activated PI3K drives stromal collagen production and accumulation. J Pathol 250: 231-242, 2020.

26. Zhu J, Zhou L, Wei B, Qian Z, Wang J, Hui H and Sun Y: miR-142-5p inhibits pancreatic cancer cell migration and invasion by targeting PIK3CA. Mol Med Rep 22: 2085-2092, 2020.

27. Cai L, Chao G, Li W, Zhu J, Li F, Qi B, Wei Y, Chen S, Zhou G, Lu X, et al: Activated CD4 ${ }^{+}$T cells-derived exosomal miR-142-3p boosts post-ischemic ventricular remodeling by activating myofibroblast. Aging (Albany NY) 12: 7380-7396, 2020.

28. Guiot J, Cambier M, Boeckx A, Henket M, Nivelles O, Gester F, Louis E, Malaise M, Dequiedt F, Louis R, et al: Macrophage-derived exosomes attenuate fibrosis in airway epithelial cells through delivery of antifibrotic miR-142-3p. Thorax 75: 870-881, 2020.

29. Wang Z, Liu Z, Fang X and Yang H: MiR-142-5p suppresses tumorigenesis by targeting PIK3CA in non-small cell lung cancer. Cell Physiol Biochem 43: 2505-2515, 2017.

30. Mei J, Zhang Y, Lu S and Wang J: Long non-coding RNA NNT-AS1 regulates proliferation, apoptosis, inflammation and airway remodeling of chronic obstructive pulmonary disease via targeting miR-582-5p/FBXO11 axis. Biomed Pharmacother 129: $110326,2020$.

31. Vuppalanchi R, Liang T, Goswami CP, Nalamasu R, Li L, Jones D, Wei R, Liu W, Sarasani V, Janga SC, et al: Relationship between differential hepatic microRNA expression and decreased hepatic cytochrome P450 3A activity in cirrhosis. PLoS One 8: e74471, 2013.

32. Yang F, Luo L, Zhu ZD, Zhou X, Wang Y, Xue J, Zhang J, Cai X, Chen ZL, Ma Q, et al: Chlorogenic acid inhibits liver fibrosis by blocking the miR-21-regulated TGF- $\beta 1 / \mathrm{Smad} 7$ signaling pathway in vitro and in vivo. Front Pharmacol 8: 929, 2017.

33. Zhou XL, Xu H, Liu ZB, Wu QC, Zhu RR and Liu JC: miR-21 promotes cardiac fibroblast-to-myofibroblast transformation and myocardial fibrosis by targeting Jagged1. J Cell Mol Med 22: 3816-3824, 2018.

34. Sun C, Tian J, Liu X and Guan G: MiR-21 promotes fibrosis and hypertrophy of ligamentum flavum in lumbar spinal canal stenosis by activating IL-6 expression. Biochem Biophys Res Commun 490: 1106-1111, 2017.

35. Conickx G, Mestdagh P, Avila Cobos F, Verhamme FM, Maes T, Vanaudenaerde BM, Seys LJ, Lahousse L, Kim RY, Hsu AC, et al: MicroRNA profiling reveals a role for MicroRNA-218-5p in the pathogenesis of chronic obstructive pulmonary disease. Am J Respir Crit Care Med 195: 43-56, 2017. 
36. Li Z, Qian R, Zhang J and Shi X: MiR-218-5p targets LHFPL3 to regulate proliferation, migration, and epithelial-mesenchymal transitions of human glioma cells. Biosci Rep 39: BSR20180879, 2019.

37. Brkić J, Dunk C, O'Brien J,Fu G, Nadeem L, Wang YL, Rosman D, Salem M, Shynlova O, Yougbaré I, et al: MicroRNA-218-5p promotes endovascular trophoblast differentiation and spiral artery remodeling. Mol Ther 26: 2189-2205, 2018.

38. Han S, Chen Y, Gao Y, Sun B and Kong Y: MicroRNA-218-5p inhibit the migration and proliferation of pterygium epithelial cells by targeting EGFR via PI3K/Akt/mTOR signaling pathway. Exp Eye Res 178: 37-45, 2019.

39. Baker Frost D, Savchenko A, Ogunleye A, Armstrong M and Feghali-Bostwick C: Elucidating the cellular mechanism for E2-induced dermal fibrosis. Arthritis Res Ther 23: 68, 2021.

40. Zhang Z, Zhu D, Zhang X, Liu Y, Wang J and Yan L: Tanshinone IIA regulates fibroblast proliferation and migration and post-surgery arthrofibrosis through the autophagy-mediated PI3K and AMPK-mTOR signaling pathway. Am J Transl Res 13: 565-584, 2021.
41. Qi L, He W, Yang J, Gao Y and Chen J: Endoscopic balloon dilation and submucosal injection of triamcinolone acetonide in the treatment of esophageal stricture: A single-center retrospective study. Exp Ther Med 16: 5248-5252, 2018.

42. Zhang Y, Wang X, Liu L, Chen JP and Fan ZN: Intramuscular injection of mitomycin $\mathrm{C}$ combined with endoscopic dilation for benign esophageal strictures. J Dig Dis 16: 370-376, 2015.

This work is licensed under a Creative Commons Attribution-NonCommercial-NoDerivatives 4.0 International (CC BY-NC-ND 4.0) License. 